\title{
Mechanisms of geometrical seismic attenuation
}

\author{
Igor B. Morozov
}

University of Saskatchewan, Department of Geological Sciences, Saskatoon, Canada

\author{
Article history \\ Received August 18, 2010; accepted February 14, 2011. \\ Subject classification: \\ Seismology: Waves and wave analysis, Surveys, measurements and monitoring, General or miscellaneous; Mathematical geophysics.
}

\section{ABSTRACT}

In several recent reports, we have explained the frequency dependence of the apparent seismic quality-factor $(Q)$ observed in many studies according to the effects of geometrical attenuation, which was defined as the zerofrequency limit of the temporal attenuation coefficient. In particular, geometrical attenuation was found to be positive for most waves traveling within the lithosphere. Here, we present three theoretical models that illustrate the origin of this geometrical attenuation, and we investigate the causes of its preferential positive values. In addition, we discuss the physical basis and limitations of both the conventional and new attenuation models. For waves in media with slowly varying properties, geometrical attenuation is caused by variations in the wavefront curvature, which can be both positive (for defocusing) and negative (for focusing). In media with velocity/density contrasts, incoherent reflectivity leads to geometrical-attenuation coefficients which are proportional to the mean squared reflectivity and are always positive. For "coherent" reflectivity, the geometrical attenuation is approximately zero, and the attenuation process can be described according to the concept of "scattering Q". However, the true meaning of this parameter is in describing the mean reflectivity within the medium, and not that of the traditional resonator quality factor known in mechanics. The general conclusion from these models is that non-zero and often positive levels of geometrical attenuation are common in realistic, heterogeneous media, both observationally and theoretically. When transformed into the conventional Q-factor form, this positive geometrical attenuation leads to $Q$ values that quickly increase with frequency. These predictions show that the positive frequency-dependent $Q$ observed in many datasets might represent artifacts of the transformations of the attenuation coefficients into $Q$.

\section{Introduction}

In several recent reports [Morozov 2008, 2009a-c, 2010a-d; hereafter referred to as M08, M09a-c, and M10a-d, respectively], it was argued that the conventional description of seismic attenuation using the quality factor of the medium (denoted as $Q$ ) relies on theoretical conjectures, analogies, and assumptions that are not sufficiently based on the fundamental principles of mechanics. In consequence, the resulting $Q$ models can be prone to spurious frequency dependences and lead to overly complex and ambiguous interpretations. One general manifestation of such excessive complexity might be in $Q$ values that are quickly increasing with frequency, which is commonly reported, particularly in coda and total-energy studies [e.g., Aki 1980]. As suggested in M09b, these increases can often be dictated by the very definition of $Q$ used in seismology, and not related to any rheological or scattering properties of the medium. Instead of using the $Q$ paradigm, we suggested returning to another well-known description, which is the attenuation coefficient in either its spatial $(\alpha)$ or temporal $(\gamma)$ forms. While removing the assumptions and uncertainties involved in the definition of $Q$, this description provides a simple and reliable basis for data analysis [M08, M10a] and offers several farreaching empirical generalizations [M10b].

The key message of the aforementioned reports was that for many wave types (short-period and long-period surface and body waves, coda, $P n, L g$, and even whole-Earth free oscillations), the observed variations of $\mathrm{Q}$ with frequency typically correspond to piecewise linear dependences of the attenuation coefficient, $\chi(f)$ (Figure 1). The intercept values of these dependences, denoted as $\left.\gamma \equiv \chi\right|_{f \rightarrow 0}$, are often positive in lithospheric measurements and correlate with tectonic types and ages of the crust (Figure 1) [M08]. The slopes of these linear $\chi(f)$ segments lead to a new, "effective» quality-factor-type measure, $Q_{e}=$ $[(d \chi / d f) / \pi]^{-1}$, which is usually frequency independent and significantly higher than the conventional $Q_{0}=Q(1 \mathrm{~Hz})$ (Figure 1). Thus, instead of the conventional pair of parameters $Q_{0}$ and $\eta$ in the power law $Q(f)=Q_{0} f^{\eta}$, the new description uses parameters $\gamma$ and $Q_{e}$ in the linear dependence:

$$
\chi(f)=\gamma+\frac{\pi}{Q_{e}} f
$$

Although also subject to some subtleties [M10a], the basic interpretation of parameters $\gamma$ and $Q_{e}$ is nevertheless 
much more straightforward than that of $Q_{0}$ and $\eta$. This interpretation can be summarized as follows:

1) The zero-frequency limit, $\gamma$ is principally responsible for the effects of the "structure», i.e., for ray bending, lithospheric reflections and conversions, multi-pathing, and scattering that is not accounted for by the background model, which is used for geometrical or other types of corrections that are performed prior to the attenuation measurements. Because of this meaning, we often refer to parameter $\gamma$ as "geometrical» [M08, M09a], although in certain cases, frequency-dependent geometrical spreading can cause complications with this terminology [M10a].

2) Parameters $\gamma$ or $Q_{e}$ describe the effects of anelastic attenuation and small-scale, random scattering. In relation to $\gamma$ above, we call these attenuation parameters «nongeometrical».

Notably, for at least short-period coda waves, $\gamma$ can be modeled from totally independent, structural information by using numerical waveform modeling [Morozov et al. 2008]. In contrast, the nongeometrical parameters are unrelated to the structure, although they can be recognized by the characteristic increase in the attenuation coefficient with frequency.

In many datasets, the observed frequency-dependent values of $Q \sim f^{\eta}$, with $\eta$ approaching and sometimes exceeding 1 , can be explained by the presence of a significant positive "geometrical attenuation", $\gamma>0$ (Figure 1) $[\mathrm{M} 08$, M10a, b]. Therefore, it appears that $\gamma>0$ might be due to some common physical properties of the lithosphere. In the present report, we offer some theoretical evidence for the potential causes of such values of $\gamma$. We show that positive

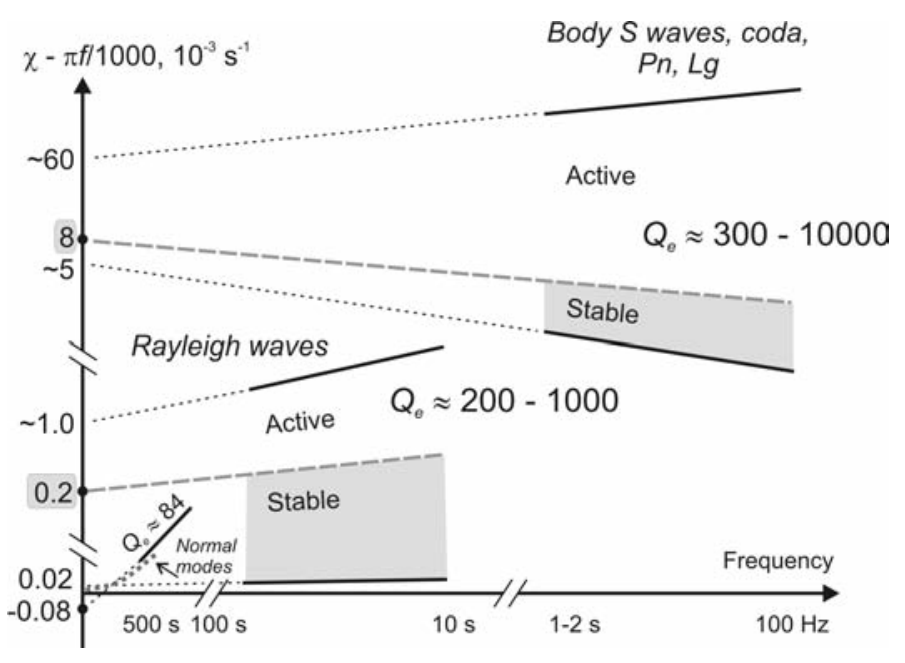

Figure 1. Summary of the observed $\chi(f)$ dependences for Rayleigh waves and short-period body, Lg, Pn, and coda waves. «Reduced» values of $(\chi-\pi f / 1000)$ are shown, so that the linear dependences corresponding to $Q_{e}=1000$ appear horizontal. Typical ranges of $Q_{e}$, and $\gamma$ levels that discriminate between the stable and active tectonic regimes are indicated by lines and gray boxes, respectively. Modified from M10b, with permission from Springer. geometrical attenuation can be caused by: 1) variations of wavefront curvatures during refraction in smoothly varying media; and 2) by incoherent reflectivity along the wavepropagation paths. In the conventional, Q-based paradigm, such values can also be attributed to a strongly frequencydependent "scattering Q", although such terminology can be misleading because of its attribution the deterministic effects of the structure to a $Q[\mathrm{M} 09 \mathrm{a}, \mathrm{M} 10 \mathrm{a}]$. However, in the third example below, we also consider a case of short-scale, "coherent» reflectivity, for which a kind of «scattering $Q$ » becomes meaningful and frequency independent.

Despite its simplicity and productive use, the model of Equation (1.1) recently met with significant criticism [Xie and Fehler 2009, Xie 2010], which even led to a special forum in Pure and Applied Geophysics [Mitchell 2010]. The critique touched upon a broad range of subjects but focused primarily on the perceived lack of a physical meaning of the expression of Equation (1.1), and particularly of its geometrical part, $\zeta$. The theoretical examples developed in Section 3 (below) answer these questions by explaining the physical rationale of the functional form of Equation (1.1) and by illustrating the physical mechanisms and approximations involved in the concepts of $\chi$ and $\gamma$. These examples also show the practical uses and limitations of the approximation of Equation (1.1). For readers interested in the fundamentals of the concept of $Q$ and in further details of this extensive debate, additional comments are given in Appendix A. Although not critical for the present report, this discussion helps to provide an understanding of the physics of the attenuation coefficient and its relation to the conventional seismic attenuation model and viscoelasticity.

\section{Apparent and intrinsic attenuation coefficients}

The observed (apparent) temporal attenuation coefficient, denoted as $\chi$ here, was heuristically inferred in M08 and M10a,b by analyzing the seismic path factor within several frequency bands:

$$
P=G_{0} \delta P, \text { where } \delta P=e^{-\chi t} .
$$

In these expressions of Equation (2.1), $P$ denotes the seismic amplitude that is corrected for the source and receiver effects, and $G_{0}$ is the reference geometrical spreading, such as $G_{0}(t)=t^{-1}$ used in many local-coda studies. Factor $\delta P$ is the residual of $P$ that remains after the geometrical correction, and $e^{-\chi t}$ represents the perturbation-theory approximation for this $\delta P$. This approximation means that $G_{0}$ is normalized so that at $t=0, P=G_{0}$, and for $t>0, \delta P$ is predicted by the scattering theory [M10a]. Equation (2.1) represents the starting point of most attenuation measurements, in which $\chi$ is directly measured from either the time-domain logarithmic decrements of the amplitudes, or from the widths of the spectral peaks near the resonances (Figure 2). 

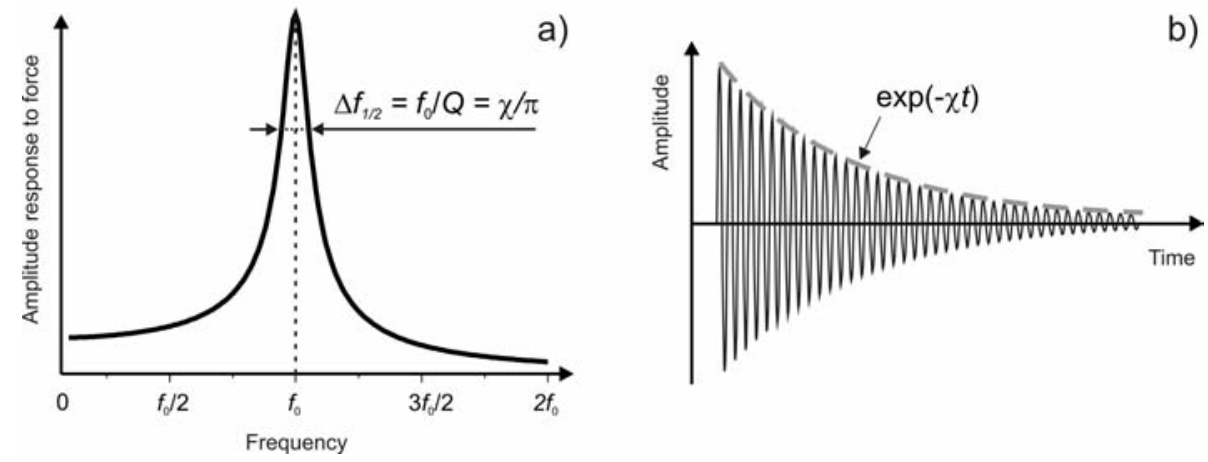

Figure 2. Definition of the attenuation coefficient, $\chi$, and the quality factor, $Q$, for an oscillatory process in (a) the frequency-domain, and (b) the timedomain. In a steady-state oscillation, the attenuation coefficient measures the width of the resonance peak at $1 / \sqrt{2}$ of the maximum amplitude, and Q measures its relative width: $\Delta f_{1 / 2}=\chi / \pi=f_{0} / Q$. An example with $Q=10$ is shown.

Unfortunately, $\chi$ is rarely studied by itself, as it is usually converted into the «apparent $Q$ » by assuming a specific form for its frequency-dependence [e.g., Aki 1980]:

$$
\chi(f)=\frac{\pi f}{Q(f)} \text {, and accordingly, } Q(f)=\frac{\pi f}{\chi(f)} .
$$

Note that $Q$ is never measured directly, but is only derived from $\chi$ by using the second expression of Equation (2.2). Although it appears only as a simple scaling, the transformation of Equation (2.2) has a deep implication: it assumes that $\chi \longrightarrow 0$ when $f \rightarrow 0$. However, this assumption is too restrictive and inaccurate in most practical cases. The transformation of Equation (2.2) assumes that attenuation occurs proportionally to the number of oscillation cycles and is meaningful only for purely oscillatory processes, such as shown in Figure 2. Nevertheless, in reality, often includes contributions from ray bending, reflectivity, multi-pathing, scattering, and other effects of the structure, for which $\left.\chi\right|_{f \rightarrow 0} \neq 0$. In such cases, the transformation of Equation (2.2) results in $Q(f)$ values that are nearly proportional to $f$, which is often observed.

To avoid the tendency of $Q$ to spuriously increase with frequency, we do not use the restrictive model for $Q(f)$ in Equation (2.2) and we view $\chi(f)$ as an arbitrary function. In empirical data analysis, it is useful to start by isolating its zerofrequency limit $\gamma$ in $\chi(f)$ [M08]:

$$
\chi(f)=\gamma+f \kappa(f) .
$$

Compared to Equation (2.2), the only difference of this form is in allowing $\gamma$ to be nonzero. For $\gamma=0$, Equations (2.2) and (2.3) are equivalent, which once again emphasizes the character of the assumption on which the approximation of Equation (2.2) is based.

The dimensionless parameter $\kappa$ in Equation (2.3) can generally be frequency dependent; however, from several data examples [M08, M09a, M10a, b] and from numerical modeling of the seismic coda [Morozov et al. 2008] and mantle Love waves, $\kappa$ turns out to be frequency independent for many wave types and frequency bands. The only waves for which the measured $\kappa$ is clearly frequency dependent are the free oscillations of the Earth, and even for these, $\kappa(f)$ appears to break into only two linear branches of the form of Equation (2.2) (Figure 1) [M10a]. For comparisons with the conventional terminology, $\kappa$ can be transformed into an «effective» quality factor $Q_{e}=\pi / \kappa$ used in Equation (1.1) [M08].

Note that the apparent $\chi$ is also closely related to the parameter $t^{\star}$ that is often used in body-wave attenuation studies [e.g., Der and Lees 1985] as $\chi=\pi f t^{\star} / t$. This parameter is usually interpreted as $Q^{-1}$ accumulated along the ray path:

$$
t^{\star}=\int_{\text {path }} Q^{-1} d t
$$

where $t$ is the propagation time. Because of its affinity to $Q^{-1}, t^{\star}$ also shows the same instability with respect to the background geometrical-spreading correction and a similar variation with frequency. For example, the values of $t^{\star}$ for body $P$-waves decrease from $\sim 1 \mathrm{~s}$ for long-period waves to $\sim 0.2 \mathrm{~s}$ for short periods [Der and Lees 1985], which may also be a spurious (apparent) effect that is related to the use of the Q-type model of attenuation. We discuss this point elsewhere in more detail. However, because the frequencydependent $Q^{-1}$ in seismology is essentially used as a proxy for $\chi$ (Equation 2.2), $\chi$ can also be represented by a path average of the corresponding «intrinsic attenuation coefficient», $\chi_{i}[\mathrm{M} 10 \mathrm{~b}]$ :

$$
\chi=\frac{1}{t} \int_{\text {path }} \chi_{i} d \tau .
$$

This new quantity combines local variations of geometrical spreading, scattering, and anelastic attenuation within the medium. Of these three factors, the anelastic attenuation is the one that definitely requires a frequencydependent $\chi_{i}$ (compare this to Equation 2.2). As argued in 
detail in M10a, the other two factors can only be separated by making additional simplifications, such as assuming a frequency-independent residual geometrical spreading. The difficulty of their separation is related to the fundamental ambiguity in the definitions of the geometrical spreading and scattering. However, in many practical cases, including the present report, separation of these quantities is not required, and $\chi_{i}$ can be treated as a single medium property.

The most important observation from Equations (2.1) and (2.5) is that for traveling waves, $\delta P$ represents a path integral, which can be rendered in either the temporal or spatial forms:

$$
\delta P=\exp \left(-\int_{\text {path }} \chi_{i} d t\right)=\exp \left(-\int_{\text {path }} \alpha_{i} d s\right),
$$

where $s$ is the ray path length, and $\alpha_{i}$ and $\chi_{i}$ are the corresponding spatial and temporal intrinsic attenuation coefficients. This shows that the variations of geometrical spreading, scattering, and attenuation have similar characters and are accumulated over the wave propagation paths. The exponential form has important general properties and similarities to ray-field, wave-field, and quantum-field mechanics.

\section{Models for geometrical attenuation}

In this section, we consider three theoretical examples that illustrate the path-integral forms (Equation 2.6) for $\chi$ and show what mechanisms can create the linear frequency dependences of $\chi$ (Equation 2.2). As we will see, the residual geometrical spreading $\gamma_{i}$ and the corresponding frequency-independent $\kappa_{i}$ occur in several end-member cases:

1) Refraction in a medium with smoothly varying velocities. This example shows that $\gamma_{i}$ is also related to the variations of wavefront curvature (i.e., to perturbations of the traditional geometrical spreading).

2) Incoherent normal-incidence reflectivity, which corresponds to large numbers of sparse reflections occurring during long propagation paths. In this case, $\gamma_{i}$ is proportional to the gradient of the acoustic impedance.

3) Short-scale reflectivity, with random but «coordinated» (alternating) reflectivity at scale lengths much shorter than the length of the incident wave. In this case, the reflectivity becomes "coherent» at $f \rightarrow 0$, and consequently $\gamma_{i}=0$. This example is studied numerically, as in Richards and Menke [1983].

All of these cases relate to the elastic processes of refraction or reflectivity, which fall under the category of «scattering», or more generally, the "geometrical» attenuation processes discussed in M08 and M10a. Our specific goal here is to illustrate the origins of the geometrical parameter $\gamma_{i}$ in theoretically tractable cases. The physics of anelastic attenuation is not discussed in these examples, and its effects are simply incorporated by the additional factor $\exp \left(-\kappa_{i} f t\right)$.

\subsection{Variations of wavefront curvature}

Dynamic ray theory [Červený 2001] illustrates the origins of the exponential form of Equation (2.6) for the attenuation coefficient. In this theory, the wave-amplitude variation is described by the ray propagator $\Pi$, which in logarithmic form is [Equation 4.4.86 in Červený 2001]:

$$
\ln \Pi(R, S)=\ln \Pi\left(R, \tilde{Q}_{N}\right)+\sum_{i=1}^{N} \Upsilon\left(\tilde{Q}_{i}, \tilde{Q}_{i-1}\right) .
$$

Here, $S$ is the source, $R$ is the receiver, $Q_{i}$ and $\tilde{Q}_{i}$ and are the incidence and emergence points at the $i$-th interface, respectively (Figure 3), and:

$$
\Upsilon\left(\tilde{Q}_{i}, \tilde{Q}_{i+1}\right)=\ln \left[\Pi\left(\tilde{Q}_{i}, Q_{i}\right) \Pi\left(Q_{i}, \tilde{Q}_{i-1}\right)\right] .
$$

In our notation (Equation 2.6), $\delta P$ corresponds to $\Pi(R, S)$, and $\Upsilon\left(\tilde{Q}_{i}, \tilde{Q}_{i-1}\right)$ is equal to $\int \chi_{i} d \tau$, where $\chi_{i}$ is the intrinsic attenuation coefficient, and the integral is taken from point $Q_{i-1}$ to $Q_{i}$ along the ray.

In the absence of interfaces and caustics, the geometrical spreading is caused by the variations in the waveform curvature (Figure 3). In dynamic ray theory, this curvature is denoted as $H$ and measured by the trace of the wavefront curvature matrix, $\mathbf{K}: H=1 / 2 \operatorname{tr} \mathbf{K}$. Matrix $\mathbf{K}$ consists of second derivatives of the travel-time field $T$ with respect to the wavefront-orthonormal coordinates $y_{k}$ [Equation 4.6.15 in Červený 2001]:

$$
\mathrm{K}_{i j}=V \frac{\partial^{2} T}{\partial y_{i} \partial y_{j}},
$$

where $V$ is the wave velocity. The wavefront curvature $H$ is related to the theoretical geometrical spreading of the ray, $G_{0} \propto L^{-1}$, by the following differential equation [Equations 4.10.28-29 in Červený 2001]:

$$
H=L^{-1} \frac{d L}{d s},
$$

where $L$ is the geometrical-spreading denominator, and $s$ is

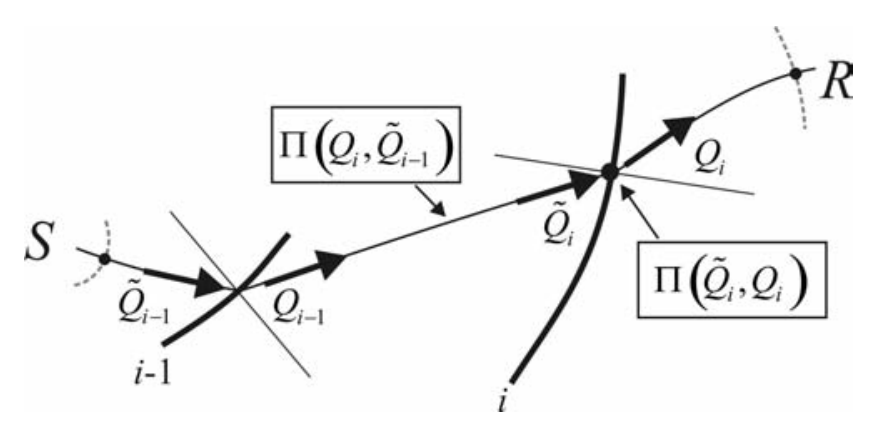

Figure 3. Ray propagator in a layered medium (Equation 3.1). Geometrical spreading is related to the ratio of the wavefront curvature (gray dashed lines) at the receiver $(\mathrm{R})$ and source $(\mathrm{S})$. 
the ray arc length. The solution to this equation relating $L(R)$ at the receiver to $L(S)$ at the source is:

$$
L(R)=L(S) \exp \left(\int_{S}^{R} H d s\right)
$$

which has the expected exponential path-integral form of Equation (2.6). Ratio $G=L(S) / L(R)$ represents the geometrical spreading factor, which is equal to $G_{0} \delta P=\exp \left(-\alpha_{i} s\right)$. In the presence of the anelastic attenuation given by parameter $\kappa_{i}$, the full path factor becomes:

$$
\delta P=\frac{1}{G_{0}} \frac{L(S)}{L(R)} \exp \left(-f \int_{S}^{R} \frac{\kappa_{i} d s}{V}\right),
$$

and by writing this expression in terms of the intrinsic spatial attenuation coefficient, $\alpha_{i}$,

$$
\delta P=\exp \left(-\int_{S}^{R} \alpha_{i} d s\right)
$$

we can see that $\alpha_{i}$ equals in this case:

$$
\alpha_{i}=H-\ln G_{0}+\frac{\kappa_{i}}{V_{i}} f
$$

with the corresponding relation for $\chi_{i}=\alpha_{i} V$. These expressions show that for smoothly refracting waves, $\alpha_{i}$ contains a frequency-independent "geometrical» $\operatorname{part}\left(H-\ln G_{0}\right)$, which is equal to the difference of the actual wavefront curvature from that predicted by the geometrical-spreading law selected as the background reference.

\subsection{Incoherent reflectivity}

To understand the relation of the in-situ attenuation coefficient to the properties of the medium, it is instructive to analyze its properties in a simple one-dimensional (1D) medium. For plane-wave propagation, the theoretical geometrical-spreading factor $G_{0}$ is equal to 1 ; however, reflections within a heterogeneous medium result in deviations from this level. As the transmission coefficients are completely described by the reflection-coefficient series, the geometrical part of the attenuation coefficient should also be related to reflectivity. Indeed, as shown below, the geometrical-attenuation coefficient is equal to half of the average squared reflection coefficient.

To begin, consider a boundary between two layers of acoustic impedances $Z_{j-1}$ and $Z_{j}$ (Figure 4). The specific expression for impedance depends on the local properties of the medium, the wave type, and the angle of its incidence on the boundary. From $[\mathrm{M} 10 \mathrm{c}]$, in the presence of attenuation, the complex-valued acoustic impedance for a $P$-wave or $S$-wave at normal incidence is:

$$
Z=\rho V\left(1+\frac{i}{2 Q_{i}}\right)
$$

where $\rho, V$, and $Q_{i}^{-1}$ are the mass density, wave velocity, and

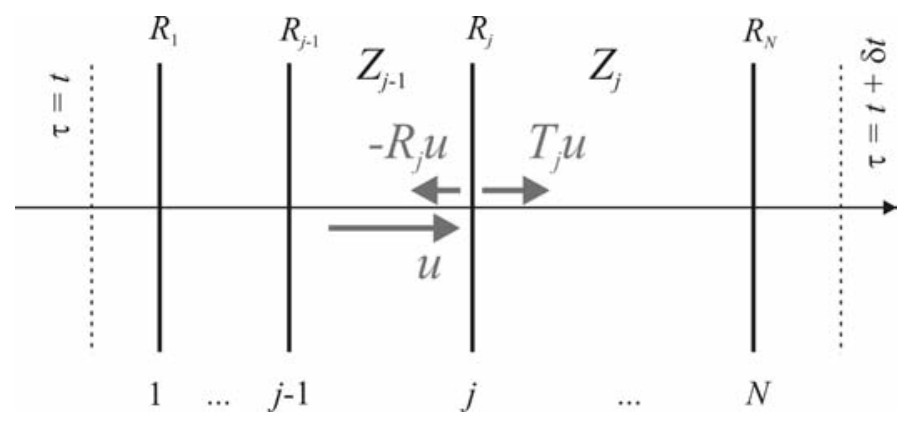

Figure 4. One-dimensional plane-wave reflection-transmission problem. Solid lines are reflectors, dashed lines are incident-wave wavefronts at times $\mathrm{t}$ and $t+\delta t$. Multiple reflections have been ignored.

parameter of anelastic attenuation, respectively. Note that although we generally argue that $Q$ cannot be considered as a medium property, this parameter is retained here for convenience of comparison to the current terminology. This use of anelastic $Q$ is possible because we are considering an otherwise uniform background, in which the geometrical spreading is accurately known, and so a single wave type is used, and therefore $Q$ appears in its specific, phenomenological sense of a plane-wave amplitude decay parameter [M09b].

Considering for simplicity the normal-incidence case, and denoting the displacement in the incident wave as $\mathrm{u}$, the displacements in the reflected and transmitted waves become $\left(-R_{i} u\right)$ and $T_{i} u$, respectively (Figure 4$)$, where, $R_{i}$ is the reflection coefficient,

$$
R_{j}=\frac{Z_{j}-Z_{j-1}}{Z_{j}+Z_{j-1}}
$$

and $T_{j}=1-R_{j}$ is the transmission coefficient,

$$
T_{j}=\frac{2 Z_{j-1}}{Z_{j}+Z_{j-1}} .
$$

The corresponding transmission coefficient for energy is:

$$
T_{E, j}=\frac{Z_{j}}{Z_{j-1}} T_{j}^{2}=\frac{4 Z_{j-1} Z_{j}}{\left(Z_{j}+Z_{j+1}\right)^{2}},
$$

and the energy reflection coefficient is equal to $R_{E, j}=1-T_{E, j}$.

For small impedance contrasts, the above coefficients are:

$$
\begin{gathered}
R_{j}=\frac{1}{2} \delta_{j}(\ln Z), \\
T_{j}=1-\frac{1}{2} \delta_{j}(\ln Z), \\
T_{E, j}=1-\frac{1}{4}\left|\delta_{j}(\ln Z)\right|^{2}=1-\left|R_{j}\right|^{2},
\end{gathered}
$$

where $\delta_{j}(X)$ denotes the contrast in quantity $X$ across the $j$-th boundary. Switching to a continuous $Z(t)$ description, the impedance contrasts over an infinitesimal propagation time 
interval $[t, t+\delta t]$ can be considered small, and therefore from Equation (3.17),

$$
\ln T_{E} \approx-\sum_{j=1}^{N}\left|R_{j}\right|^{2}=-\int_{t}^{t+s t}|r|^{2} d \tau
$$

where $r(t)$ is the root-mean square (RMS) density of reflectivity.

Equation (3.18) only gives the transmission loss caused by reflections on the boundaries passed by the wave between propagation times $t$ and $t+\delta t$. The anelastic medium attenuation over the same time interval leads to an additional energy decay:

$$
\ln T_{E} \approx-\int_{t}^{t+s t}|r|^{2} d \tau-2 f \int_{t}^{t+s t} \kappa_{i} d \tau
$$

where $\kappa_{i}$ is the non-geometrical attenuation factor.

If the transmitted waves interfere incoherently, the energy transmission coefficients combine multiplicatively over the propagation time, and therefore their logarithms are additive. For a wave that traverses $N$ boundaries in a finite propagation time $t$, the energy density $E(t)$ is (Figure 4 ):

$$
E_{N}=E_{0} \prod_{j=1}^{N} T_{E, j}=E_{0} \exp \left[\sum_{j=1}^{N} \ln T_{E, j}\right],
$$

or in terms of the continuous reflectivity function, $r(t)$ :

$$
E(t)=E(0) \exp \left\{-\int_{t=0}^{t}\left[|r|^{2}+2 \kappa_{i} f\right] d \tau\right\}
$$

This expression shows that the logarithm of the transmitted energy loss is given by a path integral,

$$
\ln E(t)-\ln E(0)=-\int_{0}^{t}\left[|r|^{2}+2 \kappa_{i} f\right] d \tau,
$$

and consequently the temporal attenuation coefficient is equal to:

$$
\chi_{i}=-\frac{1}{2} \frac{d \ln E(t)}{d t}=\frac{|r|^{2}}{2}+\kappa_{i} f
$$

The corresponding spatial attenuation coefficient is equal to $\alpha=\chi / V$ :

$$
\alpha_{i}=\frac{|r|^{2}+\kappa_{i}}{2 V}=\frac{\left|r_{\text {spatial }}\right|^{2}}{2}+\frac{\kappa_{i}}{V} f .
$$

Equations (3.23) and (3.24) also show the differences between the temporally- and spatially-veraged RMS reflectivities, which are denoted as $r$ and $r_{\text {spatial }}$, respectively.

Thus, for incoherent $1 \mathrm{D}$ acoustic-wave propagation, the geometrical-attenuation coefficient is equal to half of the corresponding path-averaged squared reflectivity. As pathaveraged properties, $\alpha$ and $\chi$ can be evaluated over finite propagation-time intervals, and therefore they can also be time dependent.

Note that when $\kappa_{i}=0$, the resulting $\alpha_{i}$ or $\chi_{i}$ are associated with geometrical attenuation, which is the zero-frequency forward scattering in this case. In the approximation considered here (normal incidence and absence of multiple reflections), these geometrical $\alpha$ or $\chi$ are independent of the frequency and the incident wavelength.

If multiple reflections are present, frequency-dependent effects (tuning) should arise even in the geometrical limit. These effects should probably have the form of resonance peaks, rather than a continuous trend with frequency. These undulations of the recorded amplitudes with frequency ("spectral scalloping») on top of the linear trend of $\chi(f)$ (Equation 2.3) are commonly observed, as shown in several data examples recently, which were reviewed in [M08, M10a, b].

\subsection{Coherent reflectivity}

The preceding example assumed incoherent interference of scattered arrivals, which occurs at all scales, but particularly when the scatterers are large and spaced at large distances compared to the incident wavelength. In this section, we consider the opposite limit, of scatterers that are small and relatively closely spaced. In this case, destructive interference of scattered waves occurs, and the attenuation coefficient shows strong frequency dependence. For simplicity, we again consider the 1-D case, in which scattering reduces to normalincidence reflectivity. Originally, this example was analyzed by Richards and Menke [1983], who demonstrated the frequencydependent effects of scattering (Figure 5) and presented them in terms of the "scattering $Q$ ». Let us briefly review this important example from a somewhat different angle, and in particular, let us look closely at the decay of the spectral amplitudes with time.

During 1D propagation, the wavefronts remain perfectly planar, and consequently the theoretical geometrical

a)

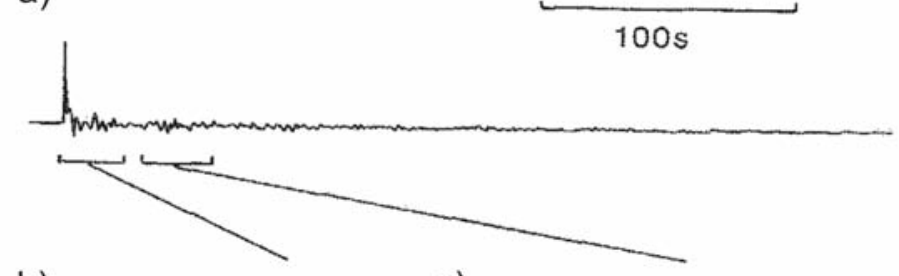

b)

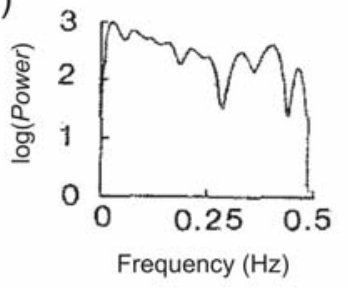

c)

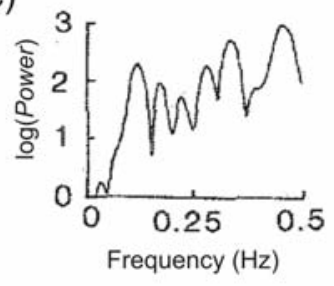

Figure 5. Transmission responses of a simulated 100-boundary sequence [modified from Richards and Menke 1983]. a) Transmitted record that resulted from a single initial pulse. b) Power spectrum of its initial part (main pulse with early forward scattering). c) Spectrum of the later forward-scattered waves. 
a)

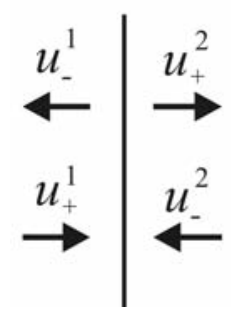

b)

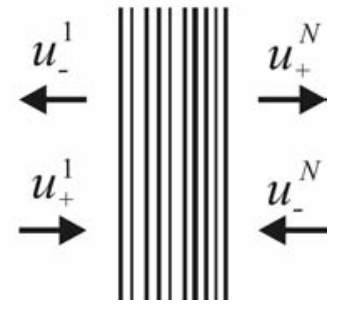

Figure 6. One-dimensional scattering problem. a) On a single boundary. b) On a random sequence of boundaries.

spreading is equal to exactly 1 . Therefore, all of the perturbations of the wavefield are due to elastic scattering on the boundaries and anelastic attenuation between them. In particular, scattering causes a part of the wave energy to reflect backward (which is often called «back scattering»), and a part of it continues propagating forwards while being delayed relative to the primary wave. This delayed part of the propagating wavefield is called «forward scattered» (Figure 6). From numerical simulations and real data, the initial wave pulse and both the back-scattered and forward-scattered waves have linear spectral variations, which increase with propagation time. Note that the senses of these variations are opposite for the initial pulse and back-scattered and forwardscattered waves, the high frequencies of which are progressively depleted and enhanced, respectively (Figure 5).

Conventionally [for example, see Richards and Menke 1983], the relative changes in the spectra (Figure 5) are viewed as proportional to the number of wavelengths traveled by the incident wave, as in Equation (2.2). The resultant spectra are fit by using approximations of the form $\exp \left(-\pi f t / Q_{s}\right)$, and the scattering is automatically viewed as analogous to the anelastic attenuation, for which the corresponding spectral amplitude decay is $\exp \left(-\pi f t / Q_{i}\right)$. However, as shown below, the proportionality to the number of wavelengths is incidental and it is only valid for coherent superposition of scattered waves. By contrast, for incoherent scattering, the attenuation tends to be independent of the incident wavelength. We therefore take a conservative view and use the Q-type parameters only for the frequencydependent part of the attenuation coefficient.

To derive the attenuation coefficient for the general case that includes both weak and strong, back scattering and forward scattering, note that the scattering problems for both the singleboundary and multi-boundary cases (Figure 6) can be described equivalently by using the scattering-matrix formulation:

$$
\left(\begin{array}{c}
u_{+}^{N} \\
u_{-}^{N}
\end{array}\right)=T_{N, 1}\left(\begin{array}{c}
u_{+}^{1} \\
u_{-}^{1}
\end{array}\right)
$$

where $N=2$ for the 1-boundary case, and $T_{N, 1}$ is the transmission matrix that relates the states on the right to those on the left in Figure 6. Here, $u$ denotes the scalar wave amplitudes, waves with subscripts ' + ' travel to the right, and those with '-' travel to the left. For a single interface, transmission matrix $T_{2,1}$ combines the coefficients of Equations (3.15) and (3.16) for forward and backward wave propagation,

$$
T_{2,1}=\frac{1}{2 Z_{2}}\left(\begin{array}{cc}
Z_{1}+Z_{2} & Z_{1}-Z_{2} \\
Z_{1}-Z_{2} & Z_{1}+Z_{2}
\end{array}\right) \approx I-\left(\begin{array}{cc}
0 & r_{2} \\
r_{2} & 0
\end{array}\right)
$$

where the second equation corresponds to the smallreflectivity approximation, $r_{2}$ is the reflectivity at the boundary, and $I$ is the identity matrix. Alternately, the amplitudes of the waves that travel away from the boundary can be related to those that are incident on it from both sides:

$$
\left(\begin{array}{l}
u_{-}^{1} \\
u_{+}^{N}
\end{array}\right)=S_{N, 1}\left(\begin{array}{l}
u_{+}^{1} \\
u_{-}^{N}
\end{array}\right) .
$$

In this expression, $\mathbf{S}_{N, 1}$ is called the scattering matrix. For $N=2$, this matrix combines the reflection and transmission coefficients in both of the propagation directions:

$$
S_{2,1}=\frac{1}{Z_{1}+Z_{2}}\left(\begin{array}{cc}
Z_{2}-Z_{1} & 2 Z_{2} \\
2 Z_{1} & Z_{1}-Z_{2}
\end{array}\right) \text {. }
$$

In the absence of anelastic attenuation, the elastic energy is preserved in the outgoing states:

$$
Z_{1}\left[\left|u_{-}^{1}\right|^{2}+\left|u_{+}^{N}\right|^{2}\right]=Z_{2}\left[\left|u_{+}^{1}\right|^{2}+\left|u_{-}^{N}\right|^{2}\right]
$$

for any $N$, and consequently the sum of the powers of backtraveling and forward-traveling waves is constant at any frequency.

For $N-1$ interfaces, matrix $\mathbf{T}_{N, 1}$ is a product of wavemode transformations on all of the boundaries:

$$
T_{N, 1}=\prod_{i=2}^{N} T_{i, i-1}\left(\begin{array}{c}
e^{i \Delta \varphi_{i}} \\
e^{-i \Delta \varphi_{i}}
\end{array}\right) \approx \exp \left[-\sum_{i=2}^{N}\left(\begin{array}{cc}
0 & r_{i} e^{i \Delta \varphi_{i}} \\
r_{i} e^{-i \Delta \varphi_{i}} & 0
\end{array}\right)\right],
$$

where $\Delta \varphi_{i}$ is the phase shift of the forward-traveling wave during its propagation in layer $i$. Let us denote the elements of this "propagator» matrix across the stack of all $N-1$ boundaries (Figure 6b) as:

$$
\mathbf{T}_{N, 1}=\left(\begin{array}{ll}
G^{++} & G^{+-} \\
G^{-+} & G^{--}
\end{array}\right) .
$$

The total reflection amplitude, $u_{-}^{1}$, can be found from the requirement that on the right-hand-side of Figure $6 \mathrm{~b}$ there should be no incoming wave traveling to the left:

$$
u_{-}^{2}=G^{-+} u_{+}^{1}+G^{--} u_{-}^{1}=0,
$$

and consequently:

$$
u_{-}^{1}=-\frac{G^{-+}}{G^{--}} u_{+}^{1} .
$$


This gives the total back-scattered amplitude. The total transmitted amplitude is therefore:

$$
u_{+}^{N}=G^{++} u_{+}^{1}+G^{+-} u_{-}^{1}=\left[G^{++}-\frac{G^{+-} G^{-+}}{G^{--}}\right] u_{+}^{1} .
$$

Equation (38a,b) can be used numerically to model the propagation of a long seismic wave through a stack of thin random layers. We use an example similar to that of Richards and Menke [1983], with 1000 layers of uncorrelated random velocities drawn from a Gaussian distribution with a mean of $3.0 \mathrm{~km} / \mathrm{s}$ and a standard deviation of $0.25 \mathrm{~km} / \mathrm{s}$. The density is assumed to be constant. Using its scale invariance, the impedance was normalized to a mean value of $Z=1$, and the same value of impedance was placed at both ends of the random sequence (Figure 7). The travel-time within each layer is taken as being equal to $1 \mathrm{~s}$, which also gives the characteristic Nyquist frequency of $f_{N}=0.5 \mathrm{~Hz}$, relative to which all of the frequencies in the propagation process can be measured.

To investigate the time "history" of scattering, the impedance time series (Figure 7) was truncated at the boundaries $N=2,3, \ldots 1000$, and the remainders of the series were closed with a layer with $Z=1$. The resulting variations of the reflected and transmitted wave intensities showed large fluctuations for the different statistical realizations of the impedance time series (Figure 8). However, after averaging these over multiple realizations, the transmitted and reflected powers showed clear and mutually complementary exponential decay (Figure 9). By measuring the logarithmic decrements of these decays, temporal attenuation coefficients $\chi$ were measured for selected normalized frequencies $f / f_{N}$ (Figure 10).

This procedure was performed for impedance contrasts spaced at regular time intervals $\Delta t_{i}=1 \mathrm{~s}$ (Figure 7) and it was also repeated for another set of random impedance variations, in which ti were randomly distributed. A lognormal distribution of $\Delta t_{i}$ was constructed so that the average $\left\langle\Delta t_{i}\right\rangle$ was also equal to $1 \mathrm{~s}$. As expected, the resulting attenuation coefficients are similar for low frequencies $f<0.3 f_{N}$. At $f>0.3 f_{N}$, the attenuation in the random- ti sequence saturates at a constant level (black line in Figure 10), although the attenuation in the regularly

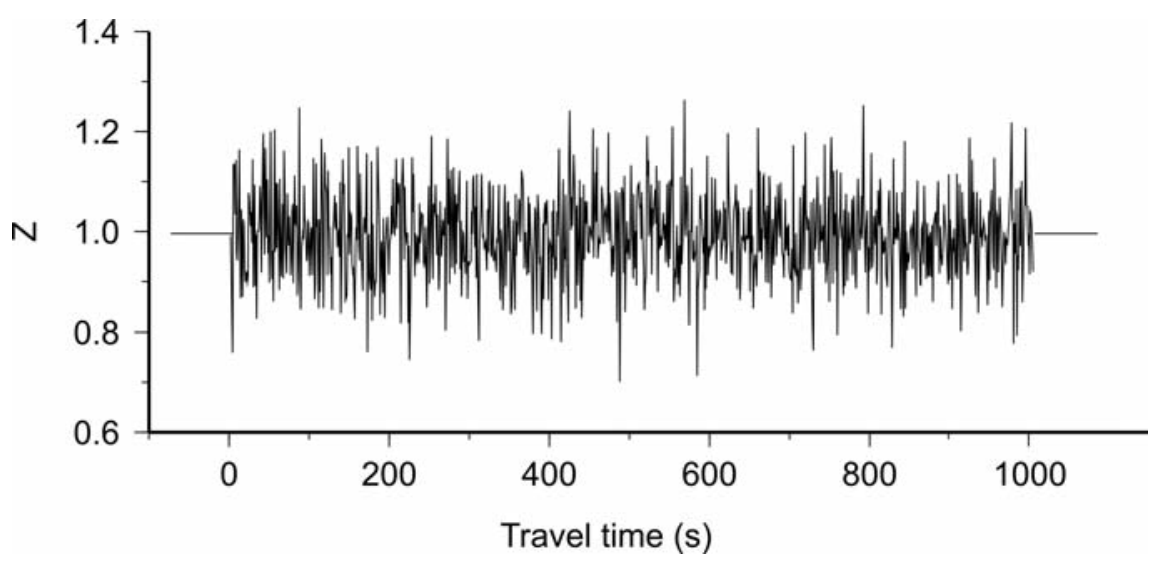

Figure 7. Random Gaussian distribution of impedance that corresponds to a mean velocity of $3.0 \mathrm{~km} / \mathrm{s}$ and a standard deviation of $0.25 \mathrm{~km} / \mathrm{s}$. The impedance is normalized to a mean value of $Z=1.0$.
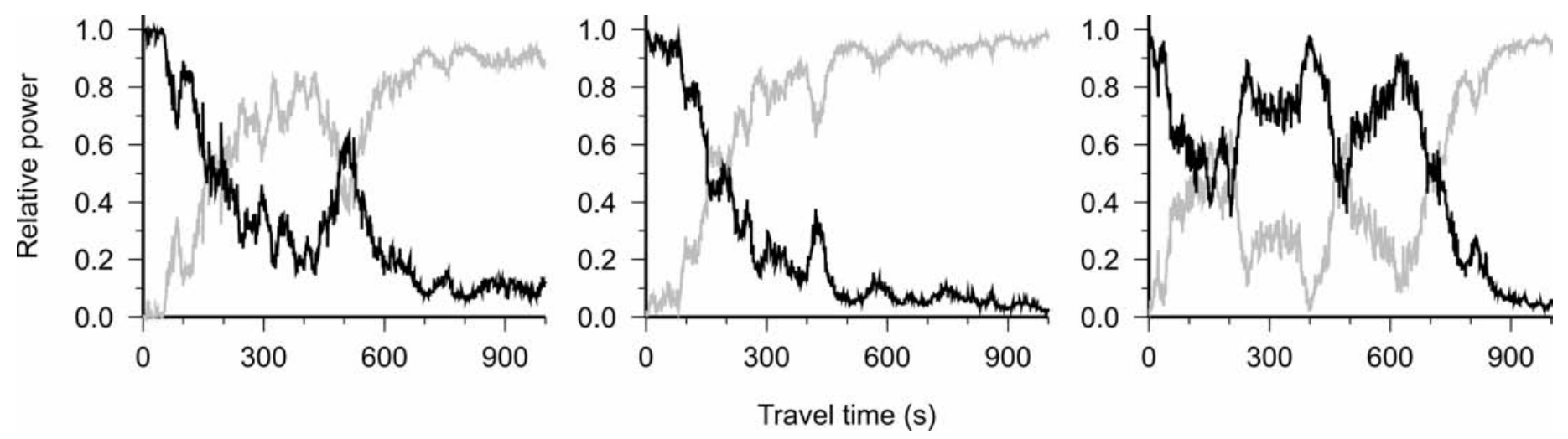

Figure 8. Wave attenuation in three statistical realizations of impedance time series (see Figure 7) for frequency $f=0.2 f_{N}$. Black and gray lines show the transmitted and reflected power, respectively. 


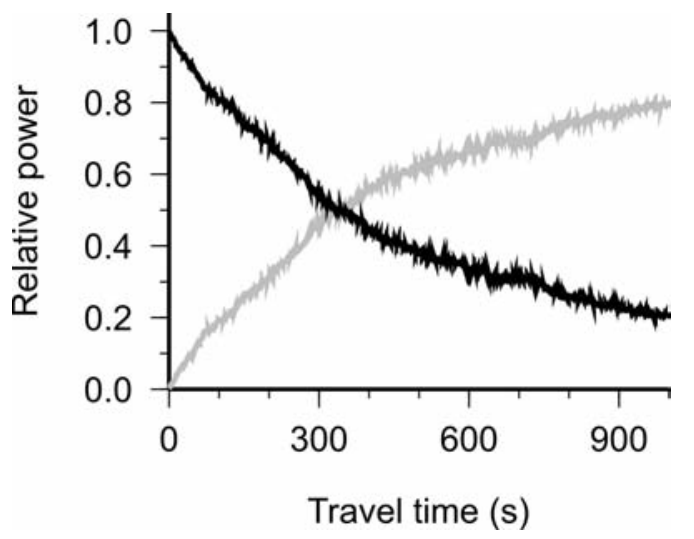

Figure 9. Transmitted (black) and reflected (gray) power averaged over 100 statistical realizations, as in Figure 8.

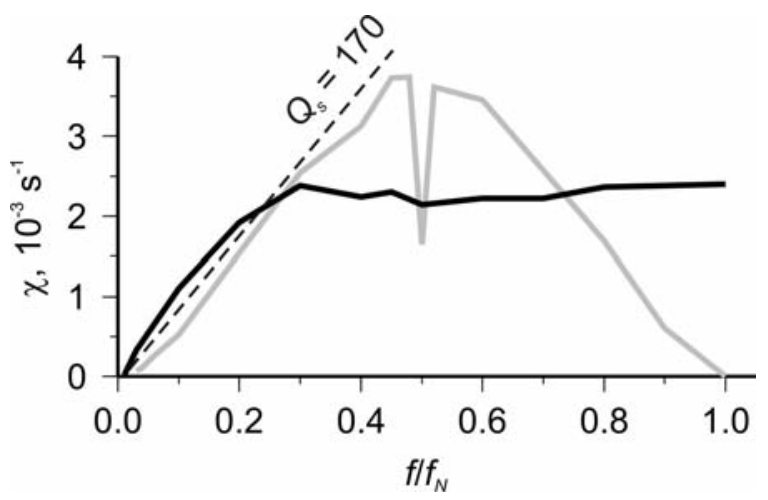

Figure 10. Frequency dependence of attenuation coefficient $\chi$ in $1 \mathrm{D}$ propagation. Gray, propagation in a sequence of layers with equal travel times; black, propagation in layers with travel-times distributed according to a log-normal distribution. Dashed line, level of «scattering Q» equal to 170.

spaced sequence continues to increase to $f \approx 0.5 f_{N}$, after which it decreases to near-zero at $f \approx f_{N}$. This pattern resembles the well known "frequency folding" effect, which is characteristic for aliasing. As can be seen, near $f \approx f_{N}$, the phases of all of the reflections superimpose equivalently to the case of $f \approx 0$, and the elastic attenuation drops to zero. Interestingly, the regularly spaced impedance series shows a very narrow «notch» at $f \approx 0.5 f_{N}$, at which the attenuation drops sharply because of the tuning of the incident wave with the reflectivity sequence (Figure 10). However, neither aliasing nor tuning are present in the more realistic random$\Delta t_{i}$ impedance series.

In summary, the general behavior of the attenuation coefficient in $1 \mathrm{D}$ random media can be described as follows:

1) At near-zero frequencies, the attenuation is low $(\chi \approx 0)$ because of the destructive interference of the impedance contrasts.

2) Up to a certain frequency $f_{0}, \chi$ increases almost linearly with $f$. In this range, "scattering $Q_{s}$ " can be meaningfully defined as $Q_{s}=\pi f / \chi$. However, this $Q_{s}$ is not a true "quality factor», but only a measure of the slope of the $\chi(f)$ dependence, which is proportional to the mean stochastic reflection amplitude. The value of $f_{0}$ might generally depend on the statistics of the distribution of layer thicknesses and is equal to $\sim 0.3 f_{N}$ in our example.

3) At frequencies $f>f_{0}$, the process of scattering becomes incoherent, and becomes frequency-independent.

The value of $f_{0}$ for a particular area might not be easy to determine; nevertheless, for an average sedimentary layering of $\sim 10$-cm thickness, $f_{0}$ can be relatively high $(\sim 40 \mathrm{kHz})$. Therefore, the entire seismological frequency band might lie within the «scattering $Q$ » regime for such layering and might exhibit a nearly frequency-independent $Q_{s}$. However, at significantly lower frequencies and longer scale lengths, the $1 \mathrm{D}$ approximation considered here breaks down because of the effects of the structure (i.e., geometrical attenuation), and the saturation of the attenuation coefficient (Figure 10), and an apparent frequency-dependent $Q_{s}$ is observed. Thus, separation of the deterministic and stochastic wavepropagation regimes is critical when considering scattering, but it cannot be done from the coda data alone.

\section{Discussion and conclusions}

The above analysis shows that in the absence of anelastic attenuation, the resulting cumulative attenuation coefficient $\chi$ is generally nonzero and depends on the refracting or reflecting structures within which the wave propagation takes place. For refraction, the zero-frequency (geometrical) attenuation coefficients can be positive (corresponding to defocusing) or negative (focusing). For incoherent reflectivity, the geometrical attenuation coefficients are always positive. For coherent reflections in which reflections of alternating polarities occur at scale lengths significantly smaller than the incident wavelength, the geometrical factor is approximately zero, and reflectivity can be described by «scattering Q». However, this last case is quite abstract, because an incoherent component should also be present in any random reflection sequence, even in the short scale length case.

Thus, the nonzero limit of $\left.\gamma \equiv \chi\right|_{f \rightarrow 0}$ is common in both the data and the theory. This limit is explained by inaccurate knowledge and variability of the background structure. In an empirical, ad-hoc interpretation that stays strictly within the paradigm of the $Q$ measurements, this limit can be attributed to a "scattering $Q$ » that increases with frequency: $Q_{s}=\pi f / \gamma$. However, this $Q$-factor terminology would ignore most of the other known information about the structure, such as the existence of velocity gradients, velocity/density contrasts, bending rays, reflections, and mode conversions. On the other hand, the concept of correctly captures these factors in the form of the geometrical spreading associated with the structure.

The two-parameter attenuation model of Equation (1.1) with constant $\gamma$ and $Q_{e}$ considered here represents only a 
first-order, perturbation-theory approximation. The theoretical examples in Section 3 indicate several limitations of this approximation, which consist in a requirement for a relatively accurate reference model $G_{0}$, a weak interactions, and finite propagation times. These examples also show how these limitations can be measured and quantified. By contrast, as shown Appendix A, the conventional Q-based paradigm has many more problems, which are difficult to assess even at the level of the basic physical theory.

For unambiguous interpretation of seismic attenuation data, it is therefore important to use the attenuationcoefficient description, in which the geometrical, scattering, and anelastic-attenuation effects are treated adequately and combined in the temporal intrinsic attenuation coefficient, $\chi_{i}$. Compared to this model, the emphasis on the frequencydependent quality-factor might lead to interpretations that are overly complex, but unrelated to the available structural information.

Acknowledgements. This study was supported by NSERC Discovery Grant RGPIN261610-03. GNU Octave software (http://www.gnu.org/ / software/octave/) was used for numerical modeling. Comments by an Associate Editor and two anonymous reviewers helped in the improvement of this manuscript.

\section{References}

Aki, K. (1980). Scattering and attenuation of shear waves in the lithosphere, J. Geophys. Res., 85, 6496-6504.

Aki, K. and P.G. Richards (2002). Quantitative Seismology, Second Ed., University Science Books, Sausalito, California.

Biot, M.A. (1962). Mechanics of deformation and acoustic propagation in porous media, J. Appl. Phys., 23, 14821498.

Bourbié, T., O. Coussy and B. Zinsiger (1987). Acoustics of Porous Media, Editions TECHNIP, France.

Carcione, J.M. (2007). Wavefields in Real Media: Wave propagation in anisotropic anelastic, porous, and electromagnetic media, Second Edition, Elsevier.

Červený, V. (2001). Seismic Ray Theory, Cambridge University Press, New York, NY, USA.

Dahlen, F. A. and J. Tromp (1998). Theoretical Global Seismology, Princeton Univ. Press, Princeton, NJ, USA.

Dalton, C.A. and G. Ekström (2006). Global models of surface wave attenuation, J. Geophys. Res., 111, B05317; doi: 10.1029/2005JB003997.

Der, Z.A. and A.C. Lees (1985). Methodologies for estimating $t^{*}(f)$ from short-period body waves and regional variations of $t^{*}(f)$ in the United States, Geophys. J. Roy. Astr. Soc., 82, 125-140.

Durek, J. and G. Ekström (1996). A radial model of anelasticity consistent with long-period surface-wave attenuation, Bull. Seismol. Soc. Am., 86, 155-158.

Dziewonski, A.M. and D.L. Anderson (1981). Preliminary
Reference Earth Model (PREM), Phys. Earth Planet. Inter., 25, 297-356.

Faul, U.H., J.D.F. Gerald and I. Jackson (2004). Shear-wave attenuation and dispersion in melt-bearing olivine polycrystals: 2. Microstructural interpretation and seismological implications, J. Geophys. Res., 109, B06202; doi: 10.1029/2003JB002407.

Kinoshita, S. (1994). Frequency-dependent attenuation of shear waves in the crust of the southern Kanto area, Japan, Bull. Seismol. Soc. Am., 84, 1387-1396.

Knopoff, L. (1964). Q, Rev. Geophys., 2, 625-660.

Liu, H.P., D.L. Anderson and H. Kanamori (1976). Velocity dispersion due to anelasticity: implications for seismology and mantle composition, Geophys. J. Roy. Astr. Soc., 47, 41-58.

Mitchell, B. (2010). Prologue and invitation to participate in a forum on the frequency dependence of seismic $Q$, Pure Appl. Geophys., 167, 1129; doi: 10.1007/ s00024010-0180-3.

Morozov, I.B. (2008). Geometrical attenuation, frequency dependence of $Q$, and the absorption band problem, Geophys. J. Int., 175, 239-252.

Morozov, I.B., C. Zhang, J.N. Duenow, E.A. Morozova and S. Smithson (2008). Frequency dependence of regional coda Q: Part I. Numerical modeling and an example from Peaceful Nuclear Explosions, Bull. Seism. Soc. Am., 98, 2615-2628; doi: 10.1785/0120080037.

Morozov, I.B. (2009a). Thirty years of confusion around «scattering Q»? Seism. Res. Lett., 80, 5-7.

Morozov, I.B. (2009b). On the use of quality factor in seismology, AGU Fall Meeting, San Francisco, CA, USA, Dec 2009, S44A-02.

Morozov, I.B. (2009c) Reply to "Comment on Thirty years of confusion around 'scattering $Q$ '?" by J. Xie and M. Fehler, Seism. Res. Lett., 80, 648-649.

Morozov, I.B. (2010a). On the causes of frequency-dependent apparent seismological Q, Pure Appl. Geophys., 167, 1131-1146; doi: 10.1007/s00024-010-0100-6.

Morozov, I.B. (2010b). Attenuation coefficients of Rayleigh and Lg waves, J. Seismol.; doi: 10.1007/s10950-0109196-5.

Morozov, I.B. (2010c). Anelastic acoustic impedance and the correspondence principle, Geophys. Prosp.; doi: 10.1111/ j.1365-2478.2010.00890.x.

Morozov, I.B. (2010d). Seismological attenuation coefficient and Q, Seismol. Res. Lett., 81, 307.

Richards, P.G., and W. Menke (1983). The apparent attenuation of a scattering medium, Bull. Seism. Soc. Am., 75, 1005-1021.

Widmer, R., G. Masters and F. Gilbert (1991). Spherically symmetric attenuation within the Earth from normal mode data, Geophys. J. Int. 104, 541-553.

Xie, J. and M. Fehler (2009). Comment on «Thirty years of 
confusion around scattering Q» by I.B. Morozov, Seism. Res. Lett., 80, 646-647.

Xie, J. (2010). Can we improve estimates of seismological Q using a new "geometrical spreading" model?, Pure Appl. Geophys., 167, 1147-1162; doi: 10.1007/s00024010-0188-8.

Corresponding author: Igor B. Morozov, University of Saskatchewan, Department of Geological Sciences, Saskatoon, Canada; email: Igor.morozov@usask.ca

C 2011 by the Istituto Nazionale di Geofisica e Vulcanologia. All rights reserved.

\section{Appendix A: Physical basis of the $\left(\gamma, Q_{e}\right)$ model}

Even if taken purely empirically, the $\left(\gamma, Q_{e}\right)$ model of Equation (1.1) leads to two important general observations:

A) The geometrical spreading is typically not known accurately enough to measure the frequency dependence of $Q$.

B) Geometrical spreading variations can and should be estimated from the data, and they represents a most valuable attribute for interpretation.

These observations were illustrated on a number of datasets from $\sim 500$-s to $\sim 100-\mathrm{Hz}$ frequency bands, and led to major reconsiderations of several interpretations [M08, M09a, b, M10a-c]. Nevertheless, Xie and Fehler [2009] and Xie [2010, hereafter X10] argued that this literally cannot be done, and presented an extensive critique of this model. Their critique follows several lines, from the functional form of Equation (1.1) and data fitting, to the physical meaning of geometrical attenuation and its relation to the viscoelastic theory and laboratory observations. At the same time, Xie and Fehler [2009] and X10 did not address the key points of A) and B) above, and they appeared to misunderstand, or misrepresent, a number of other key points of the $\chi(f)$ approach. It is therefore important to compare the conventional and new model of Equation (1.1) in light of the arguments of Xie and Fehler [2009] and X10.

\section{A.1 Data fitting and criteria for model validity}

It is known [e.g., M08, M10a, X10] that seismic attenuation data can often be fit by using either the frequency-dependent $Q(f)=Q_{0} f^{\eta}$ (i.e., $\chi(f)=\pi f^{1-\eta} / Q_{0}$ ) or linear $\chi(f)$ dependences of the type of Equation (1.1). In M08, a mapping between parameters $\left(\gamma, Q_{e}\right)$ and $\left(Q_{0}, \eta\right)$ was derived, which was also sensitive to the observation frequency band.

With the existing datasets, two-parameter frequency dependencies are probably all that can be reliably constrained, and the model of Equation (1.1) can be viewed as exploiting this by using a Maclaurin series in $f$ [M08]. The traditional power law $\chi(f)=\pi f^{1-\eta} / Q_{0}$ is another way to fit the $\chi(f)$ data with two parameters, although in a far less intuitive fashion. From the equivalence of these forms in data fitting, X10 argued that the observations of linear $\chi(f)$ dependencies «do not invalidate» the power law $Q(f)$. Indeed, Equation (A.1) cannot invalidate the power law or any other form of $Q(f)$; nevertheless, in view of its fitting the data well (indeed, often within broader frequency ranges than the power law [M08, M10a]), we can ask why a $Q(f)$ would really be required. This remains the key question, because it appears that the frequency-dependent $Q$ is only motivated by the viscoelastic theory.

The true reasons for using one or another attenuation model are not in the data fit, but in their correspondence to the physics of wave propagation [M10a]. As noted below, for viscoelasticity and $Q$, this correspondence can be seriously 
questioned. On the other hand, rigorous physical theories of waves in heterogeneous and attenuative media exist, and do not require the use of an in-situ $Q$ [Biot 1962].

\section{A.2 Measured versus assumed geometrical spreading}

The conventional practice of attenuation measurements defended by Xie and Fehler [2009] and X10 is based on the presumption that the geometrical spreading can be established by mathematical modeling and does not need to be measured. In the notation of this report, this means that $\gamma$ in Equations (2.1) and (2.3) can always be taken to be equal to zero. However, realistic geometrical spreading is practically impossible to define mathematically. Any controlled-source data section shows that «multi-pathing» (i.e., reflections, refractions, and mode conversions) is so pervasive that the wavefronts that could be followed for geometrical spreading prediction are completely absent. At the same time, despite a lack of tractable mathematical formulations, the geometrical spreading certainly exists as a physical process, and its parameters can be measured as described in M08, M10a, b. In practical observations, we only have the frequency-dependent attenuation coefficient to go by, and therefore we can only approximate the residual geometrical spreading as a frequency-independent part of the attenuation coefficient, which is given by parameter $\gamma$. For the same reason, the effects of small-scale scattering cannot be unambiguously separated from this residual geometrical spreading [M08, M10a].

\section{A.3 Physical basis of $\gamma$}

While criticizing the general idea of variable and measured geometrical spreading, X10 focused on the exponential form of the correction to $G_{0}$ :

$$
G=G_{0} \delta G \equiv G_{0} e^{-\gamma t},
$$

which arises from Equation (2.1) with $f=0$. Here, $G$ is the true geometrical spreading within the structure, $G_{0}$ is the assumed reference geometrical spreading, and we call $\delta G$ the «residual geometrical spreading» [M08, M10a]. According to $\mathrm{X} 10$, this functional form for $G$ has "no physical basis», principally because it decays too quickly at large times. However, as explained in M10a, Equation (2.1) represents a common perturbation-theory (or scattering-theory) approximation, which only means that the rate of geometrical spreading variation, $\delta G / \delta t$, is small and proportional to $G$. Similar to all perturbation models, this approximation should not be used at the limit of $t \rightarrow \infty$, in which a diffusive (also called multiple scattering) regime is established. In maybe not appreciating this point, X10 applied the $\delta G=e^{-\gamma t}$ correction to $\sim 300-\mathrm{km}$ distances [Appendices 1 and 2 in X10], whereas it was only proposed for up to 50-70 $\mathrm{km}$, and also used exaggerated values of $\gamma$. If the limit $t \rightarrow \infty$ is not considered, then both of the functional forms $\mathrm{G}_{0}$ and
$G_{0}=e^{-\gamma t}$ in Equation (A1) are equally acceptable, because $G_{0}$ itself is also only an ad-hoc, or reference, approximation for the geometrical spreading. Other forms of geometricalspreading corrections were also proposed in M10a, with the exponential model of Equation (A1) preferred because of its useful roots in the scattering approximation.

It is clearly impossible to disprove the validity of the approximation of Equation (A1), because the realistic baseline $\mathrm{G}_{0}$ has neither a unique functional form nor a definite physical meaning. In Appendix 2 of X10, an example was offered of a «physically meaningful» geometrical spreading of a wavefront spreading in a $2 \mathrm{D}$ structure with a linear velocity gradient. However, this derivation is not correct, because it assumes that the wavefront remains cylindrical in shape while propagating at different speeds in different directions. This once again illustrates the fundamental difficulty of purely mathematical approaches to geometrical spreading.

\section{A.4 Accuracy of perturbation-theory approximation}

Many studies have shown that the theoretical geometrical-spreading approximation $G_{0}$ is often inaccurate and affects the attenuation measurements, which is known as the "trade-off» of $Q$ with the assumed model geometrical spreading [e.g., Kinoshita 1994]. In M08 and related reports, this concept of a model trade-off was viewed as not acceptable, and the accuracy of $G_{0}$ was tested quantitatively using the ansatz of Equation (1.1) as a single-parameter generalization of the conventional $G_{0}$. The data showed that $\gamma$ were nonzero in most cases, and also that the entire frequency-dependent part of $Q$ can be absorbed by a correction in $\gamma$. This correction also increases the values of $Q$ (from $Q_{0}$ to $Q_{e}$ ) by as much as $\sim 20-30$-fold [M08, M10a]. Thus, the zero-order approximation $G_{0}$ is clearly insufficiently accurate for measuring the in-situ $Q$.

The next important question is whether the first-order correction of Equation (1.1) to $G_{0}$ is accurate enough. X10 correctly noted that from some datasets, $|\gamma|$ values turn out to be too large and to violate the perturbation-theory criterion $t|\gamma|<<1$, where $t$ is the characteristic observation time $[\mathrm{M} 10 \mathrm{a}]$. However, from such large values, X10 made a paradoxical conclusion that $G_{0}$ (i.e., set $\gamma=0$ ) should therefore be used and that the attenuation measurements "cannot be improved» by a better geometrical spreading model. On the contrary, large $|\gamma|$ only means that $G_{0}$ is too inaccurate, and a correction is required. With the use of a more accurate $G_{0}$, such as the numeric models of M10a, the values of $\gamma$ would reduce, and the approximation of Equation (1.1) would enter the range of its formal validity.

Finally, $Q$ represents only a second-order effect that can only be constrained when both $G_{0}$ and the residual geometrical spreading $(\gamma)$ are accounted for. It appears that, realistically, this can only be done based on the frequency dependence of $\chi$, i.e., by measuring $Q_{e}$ from the spectral 

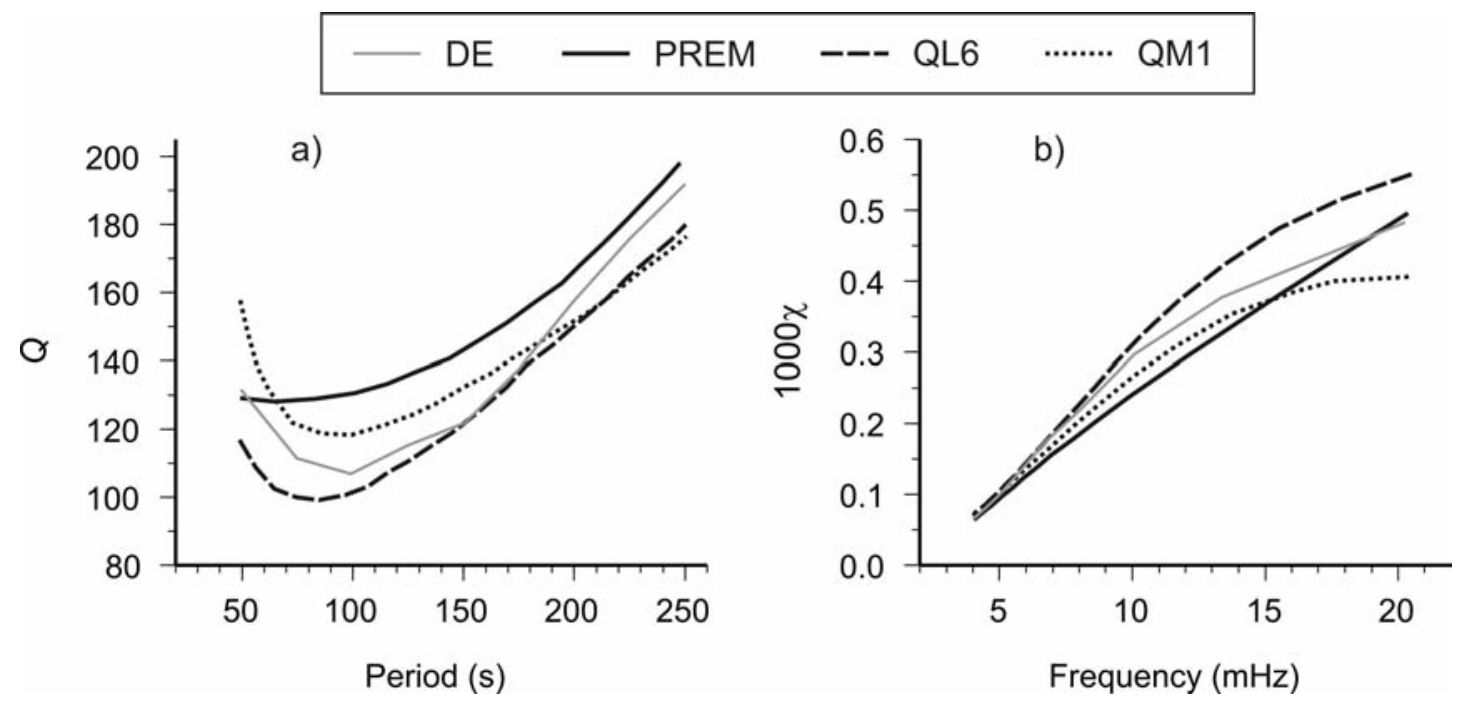

Figure A1. Apparent attenuation for four spherically symmetric global Rayleigh-wave models. a) In $Q(f)$ form. b) In $\chi(f)$ form. DE, [Dalton and Ekström 2006]; PREM, [Dziewonski and Anderson 1981]; QL6, [Durek and Ekström 1996]; and QM1, [Widmer et al. 1991]. Note that $(f)$ for PREM is near linear across the entire 50-250-s period band.

slopes of seismic amplitudes. This can only be done by assuming that the residual geometrical spreading is frequency independent [M10a].

\section{A.5 Monotony of apparent $Q(f)$ dependencies}

In a peculiar, but instructive, argument that deserves some discussion, X10 found a "fundamental contradiction» of the $\chi(f)$ model of Equation (1.1) in the fact that it only predicts apparent $Q=\pi f / \chi$ values monotonously varying with frequency. X10 pointed out that among the four major longperiod Rayleigh-wave models, only Dziewonski and Anderson [1981] showed a monotonous increase in the apparent $Q$ with $f$ (Figure A1a). However, all four models cannot be correct simultaneously, and they differ among themselves to about the same extent as from Dziewonski and Anderson [1981]. Therefore, all of these models do not have to comply with the model of Equation (1.1). At the same time, note that model PREM shows an almost perfectly linear $\chi(f)$ dependence within the entire frequency band (Figure A1b).

As Figure 10 shows, $\chi$, and even more so $Q$, are not required to vary monotonously with frequency in the model of Equation (1.1). Values $\gamma$ and $Q_{e}$ in Equations (1.1) and (2.3) are apparent quantities, and their constancy is just an empirical observation for certain wave types and frequency bands [M08]. At the same time, it is certainly remarkable that these quantities stay nearly constant within the same wave types [M10b and Section 3, present report].

\section{A.6 Laboratory observations of frequency-dependent $Q$}

An important argument in favor of a frequencydependent $Q$ and implicitly against the attenuationcoefficient model of Equation (1.1) comes from laboratory measurements using rock samples [X10]. Indeed, many laboratory observations at seismological frequencies, such as by Faul et al. [2004], yield values of $Q$ that increase with frequency. Nevertheless, it should not be thought that geometrical spreading does not exist in laboratory experiments, or that these measurements are not affected by elastic structural effects. Quite the opposite: the transformation of the measured quantities, which are the resonance-peak widths or strain-stress phase delays, into the inferred "material $Q$ » is most complex for laboratory data and relies on the most intricate models and numerous corrections [Bourbié et al. 1987]. These models have pronounced effects on the elastic limit (see Section 3) and have the role of geometrical spreading. The examples in Section 3 of the present report illustrate three types of such effects quantitatively.

\section{A.7 Physical basis of conventional attenuation models}

Ironically, the physical basis of the conventional geometrical spreading and $Q$ models (such as $G_{0} \propto t^{-V}$ and $Q=$ $=Q_{0} f^{\eta}$, respectively) is much more difficult to establish than that of the perturbation-theory formulae of Equations (1.1) and (2.3). Both of these models only arise in overly simplified theories, such as approximations of the Earth lithosphere as a uniform and isotropic half-space with a flat boundary that are commonly used in local-coda studies [e.g., Aki 1980]. The $Q_{0} f^{n}$ power law appears to be suggested by the elementary «equivalent linear solid» models [e.g., Carcione 2007], although at the same time, multiple solids are usually superimposed to allow almost arbitrary frequency dependencies of $Q$ [Liu et al. 1976]. The only definite constraint on possible $\chi(f)=\pi f / Q$ dependencies from the power-law $Q$ model consists in $\left.\chi\right|_{f \rightarrow 0}=0$. However, such a constraint is not physically justified and it is commonly 
violated in observations [M08, M09a, M10a,b].

It appears that the reason for the popularity of $Q$ in attenuation models is not in its physical validity, but in its simplicity, flexibility, and practical convenience. Once we postulate that virtually the same quantity, namely $Q^{-1}$, exists as both the material and observed (apparent) property, the theory becomes greatly simplified. The correspondence principle [Aki and Richards 2002] allows the treating of the in-situ $Q^{-1} / 2$ as a complex argument of the velocity, which dramatically simplifies modeling and inversion. The frequency dependence of the in-situ $Q$ provides a very flexible parameterization that allows the fitting and modeling of the data across broad frequency bands. However, all of this is achieved by departing from the mechanical description of the medium, which means that such a $Q$ might be no more than a heuristic mathematical model.

\section{A.8 In-situ $Q$ and viscoelasticity}

The viscoelastic theory is often used for implicit conceptual support for a frequency-dependent in-situ $Q$ of the propagating medium [X10]. This is a very extensive subject that cannot be fully addressed here; however, two observations supporting this were made in M09b. First, note that the quality factor was introduced in seismology from an intuitive analogy with acoustic or mechanical resonators [Knopoff 1964], alhough it is still not the type of quantity that can be easily associated with a point within the medium. The difficulty in the definition of a "material Q" can be seen as there are many factors that are responsible for elastic-energy dissipation, such as rock-matrix properties, grain shapes, pore volumes, shapes and connectivity, fractures, fluids, and various dielectric and piezoelectric properties. Most of these factors are unrelated to the elastic moduli and cannot be grouped together into the only two values of $Q_{K}$ and $Q_{\mu}$ used in traditional seismic viscoelasticity. Thus, the viscoelastic model is far too simple to describe the real Earth materials.

Secondly, the viscoelastic model is also too general and permissive. It only reproduces the formal convolutional relationships between the strain and stress within a wave, so it does not constrain the mechanism of energy dissipation. However, nearly any linear process can be presented as «viscoelastic» in this sense. For example, ordinary Newtonian mechanics can be described by convolutional integrals that relate the particle position, $x(t)$, to the force-rate history, $\dot{f}(t)$ :

$$
x(t)=\int_{-\infty}^{t} J(t-\tau) \dot{f}(\tau) d \tau,
$$

where $J(t)$ can be called the «delayed compliance function»:

$$
J(t)=\frac{\theta(t)}{2 m} t^{2},
$$

where $m$ is the mass, and $\theta(t)$ is the Heavyside step function. However, such a picture does not replace Newton's second law, $\ddot{x}(t)=f(t) / m$, and should not be interpreted literally, as some "memory" inherent in particle motion. Note the similarity of Equation (A2) to the viscoelastic relation of the strain, $\varepsilon$, to stress-rate history, $\dot{\sigma}$ [Dahlen and Tromp 1998]:

$$
\varepsilon(t)=\int_{-\infty}^{t} J(t-\tau) \dot{\sigma}(\tau) d \tau .
$$

Another fundamental problem in the application of the viscoelasticity to seismology is the absence of a unique definition for the elastic energy [e.g., Carcione 2007]. This leads to the absence of the traditional Hamiltonian dynamic principle, which is standard in mechanics. Among its practical manifestations, this problem leads, for example, to an incorrect complex argument of the acoustic impedance in the presence of attenuation [M10c]. 